\title{
Brain MRI literature review for interdisciplinary studies
}

\author{
Humera Tariq ${ }^{1}$, Aqil Burney² \\ 1. Department of Computer Science, University of Karachi, Karachi, Pakistan. 2. College of Computer Science and \\ Information Systems (CCSIS), Institute of Business Management (IoBM), Karachi, Pakistan.
}

Correspondence: Humera Tariq. Address: Department of Computer Science (UBIT Building), University of Karachi, Main University Road, Karachi-75270. Sindh, Pakistan. E-mail: humera@uok.edu.pk

Received: September 30, 2014

Accepted: October 27, 2014

Online Published: November 11, 2014

DOI : $10.5430 /$ jbgc.v4n4p41

URL: http://dx.doi.org/10.5430/jbgc.v4n4p41

\begin{abstract}
Computer science individuals, biomedical engineers and IT professionals require foundation knowledge about brain anatomical structures and physical sciences especially when it comes to research. The objective in this paper is to develop basic understanding of brain anatomical structure to be used for magnetic resonance imaging (MRI) and image processing along with necessary foundations to cultivate interdisciplinary research. The review incorporates the glorious history of MRI along with its marvelous mathematical contribution. This contribution makes the measurement of proton density possible which is then transformed into two dimensional magnetic resonance images for visualization and various brain related computer aided diagnostics and treatment. Spin physics, Magnetic field Precession, Larmor frequency, Bloch equation and Fourier Transform are the building blocks of magnetic resonance imaging. All the foundations which are compulsory and must be known at first sight to brain MRI are made available in this review.
\end{abstract}

\section{Key words}

Magnetic resonance imaging, Brain MRI, Magnetic precession, Larmor frequency, Bloch equation, Fourier transform

\section{I ntroduction}

Magnetic resonance imaging (MRI) is a sophisticated clinical and analytical tool for neuroscience researchers and surgeons to investigate brain. A typical brain MR study of a patient involves analyzing brain volume in three cross sections namely coronal, sagittal and axial. Each cross section further contains 10 to 30 or even more 2D slices and hence efficient storage and computational techniques are required to handle, analyze and process MRI data. Figure 1 shows the three anatomical planes, the top left head demonstrates the axial or transverse plane (xz-plane) which is parallel to ground, the view runs from head to feet and separates the superior from the inferior. The top right head is shown with coronal plane (xy-plane) which is perpendicular to the ground; the view runs from front to back and separates the ventral from the dorsal. The bottom head in Figure 1 shows sagittal plane (yz-plane) which is also perpendicular to the plane but the view in this case runs from left to right. One can think of MR data as a stack of 2D images acquired in 3D space while a person walking with camera along any one of three spatial dimensions. The camera analogy for an axial view is thus bird's eye view, for a coronal view it is to move forward and backward along z-direction while Sagittal is to pan camera from left to right along $\mathrm{x}$-axis. It is very often to use term slice for an image representing either axial, coronal or sagittal view of brain. A slice is just like a 2D image stored in matrix of size $\mathrm{M} \times \mathrm{N}$. The smallest unit of slice is voxel i.e. volumetric pixel with certain 
dimensions say $2 \mathrm{~mm} \times 2 \mathrm{~mm} \times 3 \mathrm{~mm}$ as shown in Figure 2. But if a person is lying on MRI bed, z-axis then becomes upward. A researcher needs to remember following analogy for its ease: Axial plane corresponds to XZ Plane, Coronal plane corresponds to XY plane and Sagittal plane corresponds to YZ plane. The remarkable MRI imaging technology is a breakthrough in the history of Medical Sciences and is a consequence of contribution by many Geek researchers and inventors of 17th, 18th and 19th Century. The first Nobel Prize on the way to successful NMR was received by Isidor Isaac Rabi in Physics in 1944 for measuring the properties of atomic nuclei. Though the discovery of Nuclear Magnetic Resonance i.e. NMR (former name of MRI) was led in year 1946 by Bloch and Purcell ${ }^{[1,2]}$ independently who also share Nobel Prize for this marvelous discovery in 1952, its true foundation was laid in 1974 when Paul C. Lauterbur, a Professor of Chemistry and Radiology, from New York University and Peter Mansfield, a physicist at University of Nottingham, independently, discovered the spatial localization of NMR signal to produce 2D images. Soon in 1975, Richard Ernst made use of Fourier Transform instead of Lauterbur's back-projection for rapid reconstruction of 2D image from NMR signals. Readers are encouraged to read the vivid history of MRI ${ }^{[3]}$ as this exemplary pioneer work related to MRI has led to five Nobel Prizes.

Figure 1. Anatomical planes. Oblique view of the head showing standard anatomic orientation of a coronal, a sagittal and an axial (or transverse) plane. Retrieved from ${ }^{[4]}$.

Figure 2. Voxel and slice in 3D MRI data.
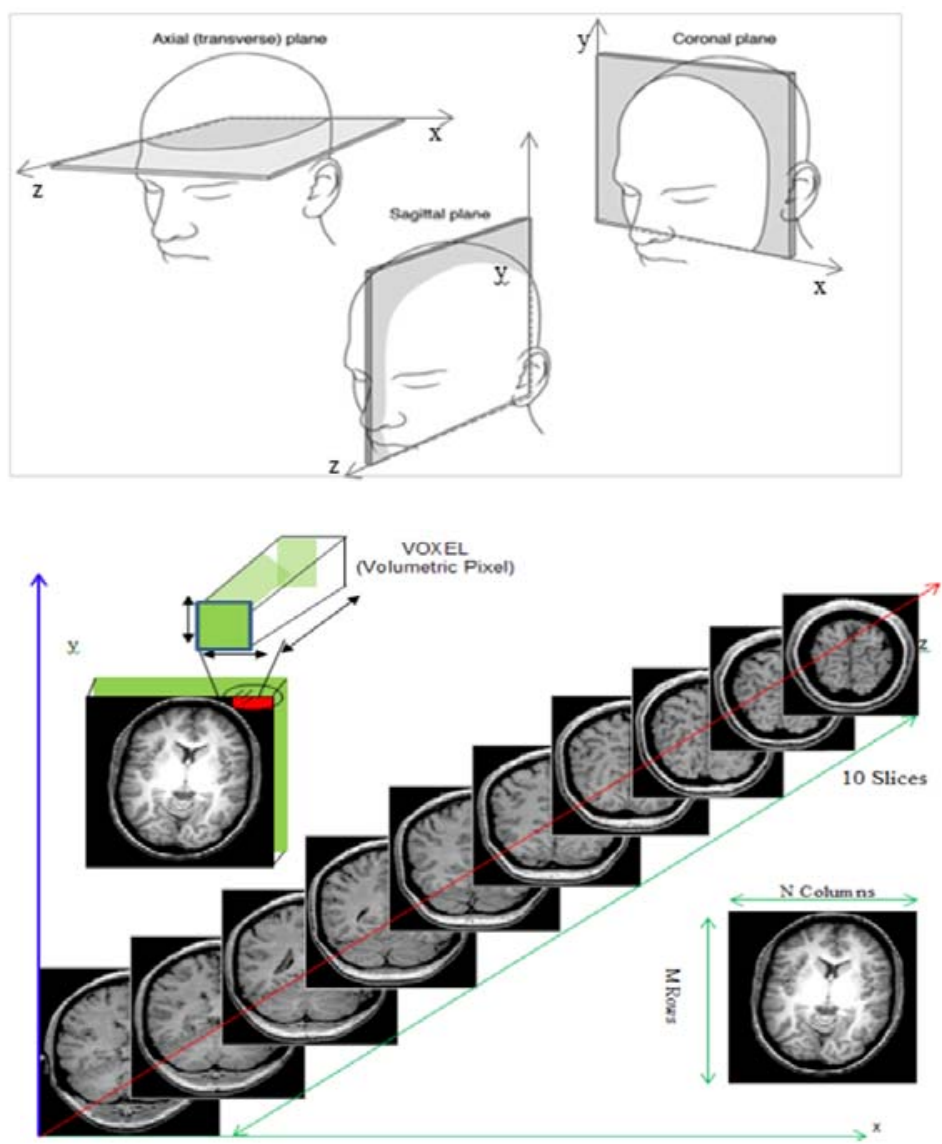

The first NMR scan through human head was first reported by a team, under the supervision of Hugh Clow and Ian R. Young ${ }^{[5]}$ at EMI Central research laboratories in England. Sir Godfrey N. Hounsfield who received the Nobel Prize "for the development of computer assisted tomography", jointly with Allan M. Cormack, describes NMR procedure as tissue detection followed by image reconstruction ${ }^{[6]}$. In his comparisons of NMR with Computerized Tomography (CT), Sir Godfrey Hounsfield clearly stated that: (1) CT mainly detects one variable-density to differentiate among tissues while MRI measures multiple spin relaxation time (T1) based on the fact that protons in different tissues return to their equilibrium state at different rates (i.e., they have different T1 times); (2) Discrimination of Fat with tissues in CT is far better than in MRI; (3) Picture Resolution in CT is considerably better than MRI; (4) Scanning time in CT is comparatively shorter than in MRI. He emphasize on the need to improve the speed and sensitivity of MRI procedure and also highlights that it is not clear enough that what parameters are measured by MRI. Sir Godfrey Hounsfield concluded that CT and 
NMR are two complementary techniques for soft tissue imaging and one should not look at them as potential competitors. According to Sir Godfrey Hounsfield, MRI provides information about chemical composition of the tissue and CT provides means to visualize tissues position and shape. Figure 3(a) shows the picture of first scanned brain using CT on laboratory machine. This brain is a preserved specimen provided by a local hospital museum. Figure 3 (b) is the brain scan of a woman with suspected brain lesion whose details are visible in scan as a dark circular cyst. Figure 3 (c) shows a closer look at first brain NMR image by Ian R. Young.
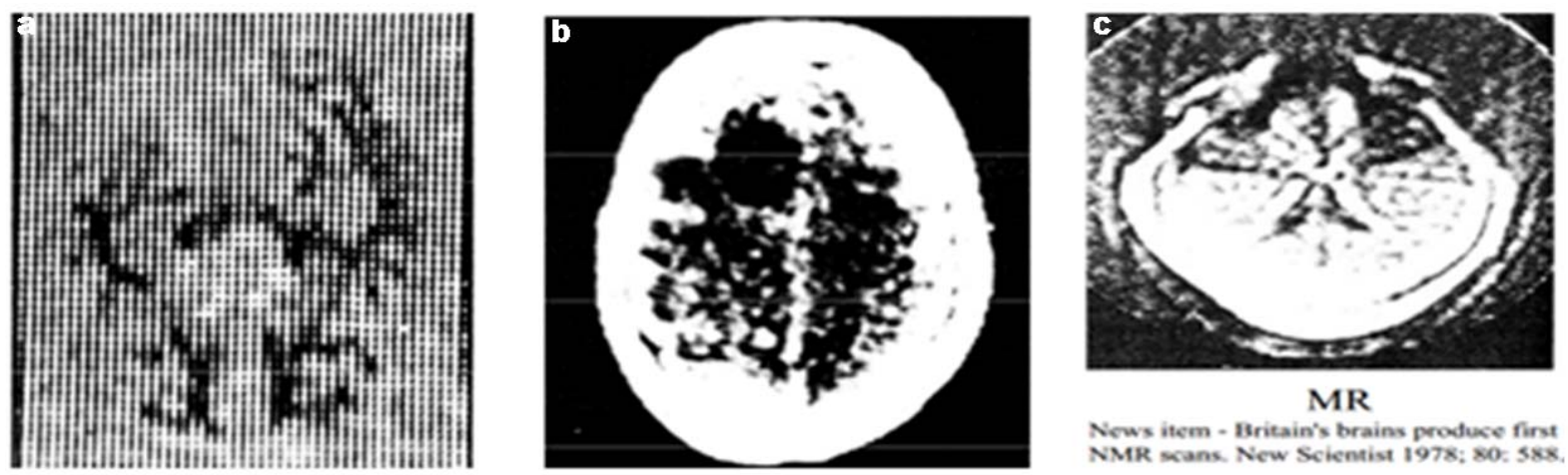

Figure 3. (a) First CT Scan of Brain ${ }^{[6]}$; (b) First CT brain Scan suspected with brain lesion ${ }^{[6]}$; (c) First Ever Human Head MR Image ${ }^{[5]}$.

\section{Human brain}

Human Central Nervous System (CNS) mainly consists of brain and spinal cord. The brain in turn consists of around one hundred billion $10^{10}$ neuron cells where each cell is believed to have two main types of matters namely gray matter and white matter. Gray matter is responsible for all processing as it contains most of cell bodies (nucleus and cytoplasm combinely called as Perikaryon) while white matter is supposed to comprised of Axons as shown in Figure 4(a). The axons of a neuron through its terminal buttons interact with dendrites of another neuron over synaptic gap (a junction where Axon meets Dendrite) and thus is responsible for information exchange between gray matter cells, from gray matter to other parts of body and towards gray matter from different body parts. In effect axons are message transmitters while dendrites are message receivers. An important difference between gray matter and white matter is myelin sheath which covers axons and are absent in gray matter neurons. Figure 4(a) is only a model to mimic neuron functionality and does not represent the actual picture of brain. To have an idea about actual picture of brain the frontal and transverse view is shown in Figure 4(b) where in cerebrum gray matter surrounds white matter while in spinal cord the white matter surrounds the gray matter.

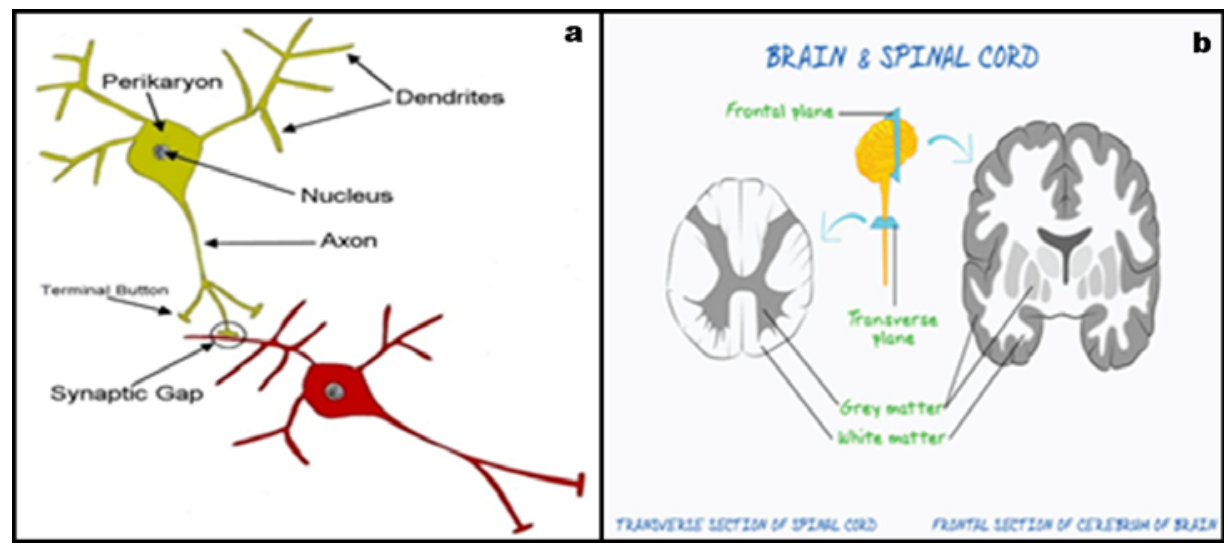

Figure 4. (a) Two interacting Neurons inside brain where Axon mimics white matter while Perikaryon mimics Gray Matter Retrieved from ${ }^{[7]}$; (b) Transverse section of spinal cord and Frontal section of cerebrum Retrieved from ${ }^{[8]}$. 
The brain sits inside hard skull or cranium along with Meninges for its protection, see Figure 5(a). The figure also shows that between our scalp (skin) and brain more appropriately cerebrum there lie tissues that PAD the brain (Pia; Arachnoid; Dura). These layers are collectively named as Meninges and are shown in Figure 5(b). In MRI scans meninges along with in-between tissue layers can be seen having a round thick structure around cerebrum in distinct contrast. The separation of these meninges from cerebrum is a frequent preprocessing step in MRI image processing. In MRI literature the process of extraction of cerebrum region ${ }^{[9]}$ is also named as head segmentation ${ }^{[10]}$ or skull stripping ${ }^{[11]}$. Tsai $\mathrm{C}^{[9]}$ uses accumulated histogram of all brain slices of volume under consideration followed by morphological operations dilation and erosion (collectively called opening) to isolate cerebrum from bone and soft tissues. This implicitly removes background noise using background threshold. In addition to skull and Meninges there exists another protective layer in the form of liquid cushion called cerebrospinal fluid (CSF). For readers ease we have built a hierarchy to summarize the vocabulary introduced so far in Figure 6.

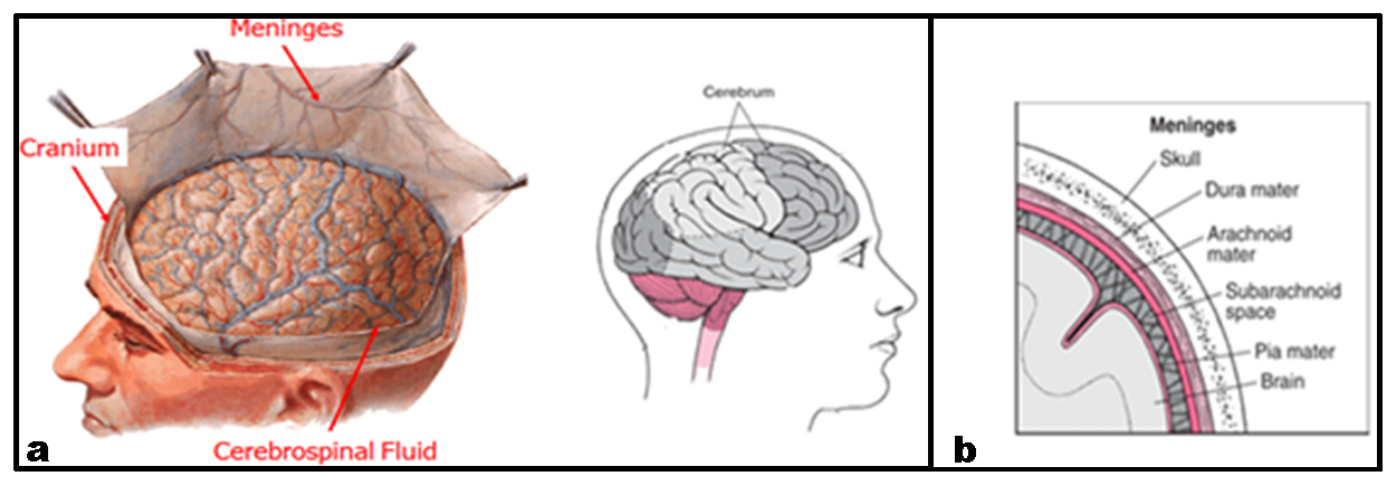

Figure 5. (a) Protection mechanism around brain ${ }^{[12]}$; (b) Illustration of tissue layers between scalp and brain ${ }^{[13]}$.

Figure 6. Central Nervous System (CNS) hierarchical diagram

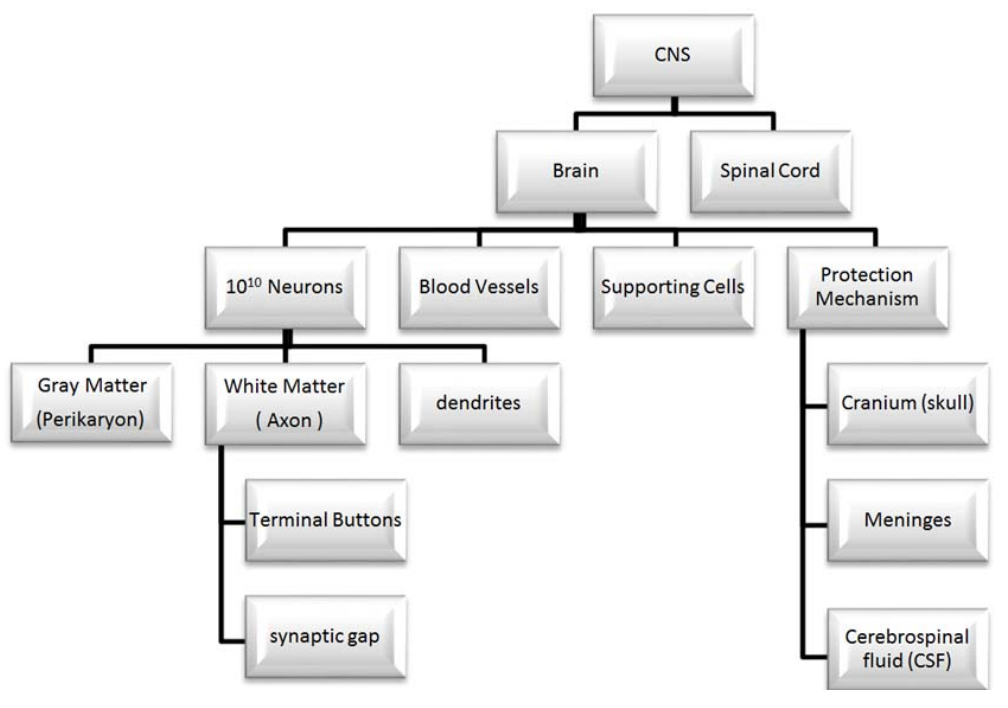

\section{I maging modalities}

Imaging Modalities means the use of X-rays, CT, Ultrasound and MRI to visualize, analyze and investigate anatomical structures. These imaging modalities are surprisingly great and led the foundation of many noble prizes in the field of physics. Wilhelm Conrad Roentgen received the very first noble prize for his remarkable invention of x-ray imaging in $1901^{[14]}$. X-rays works on the principle of ionization energy and its production needs special apparatus like cathode ray tube. The human body consists of hard bones, muscles and various soft tissues so when X-rays pass through these structures it scatter and attenuates in different direction due to different absorption characteristics. The conventional method to convert x-rays into a digital image are: (1) Film Digitization; (2) Latent to visible image conversion; (3) Use of 
fluorescent screen with CCD or CMOS arrays, where CCD stands for Charged Coupled Devices and CMOS stands for Complementary metal oxide semiconductors ${ }^{[15]}$. Tomography, (Tomo means slice; graphy means picture) by definition is slice by slice imaging of an object. A CT machine is based on spiral scanning mechanism where the scan is made by rotating an X-ray beam around the patient body. Ultrasound machines uses echoes of high frequency sound waves for image formation whereas MRI uses electromagnetic (EM) radio frequency pulses to produce anatomical images, which have very low frequency and energy in contrast to high energy X-Rays and thus MRI offers less radiation hazards in treatment and diagnostic of severe diseases.

\section{Quantum physics}

To understand image formation via MRI technology, it is important to have a cryptic look at core Quantum physics concepts related to MRI. These concepts include Proton Spin, Spin Control, Magnetization, Flipping Magnetization (a.k.a. RF Excitation) and Relaxation. Most of the discussion is studied from ${ }^{[16]}$.

\subsection{Proton spin}

Before studying MRI literature what one knows was: that electron orbits around nucleus and proton and neutron resides inside it. But according to most basic fact of quantum physics nucleus spins on its own axis just like earth spins. This means that proton and neutron are also in continuous spin state with nucleus. Protons thus imitate the nature of magnets having its own north and south poles as shown in Figure 7(a). In the absence of an external magnetic field $\mathbf{B}$ the magnetic moment $\boldsymbol{\mu}$ of individual protons are randomly oriented so no "net magnetic dipole" $\mathbf{m}$ and no "net magnetization" $\mathbf{M}$ is observed, as shown in Figure 7(b). We can say that all spins experiences zero energy: $\boldsymbol{B}=0 ; \boldsymbol{m}=\langle\boldsymbol{\mu}>=0 ; \boldsymbol{M}(\mathrm{t})=0 ; \boldsymbol{E}=0$. Millions of protons of human body subject to MRI in the presence of applied magnetic field $\mathbf{B}$ experiences a turning force torque on them and hence spin about center of mass with angular momentum $\mathbf{J}$ and magnetic moment $\boldsymbol{\mu}$. The ratio of magnetic moment to angular moment is a constant depending on type of nuclei and is known as gyromagnetic ratio $\gamma$. As an example the gyromagnetic ratio of proton is $4.26 \times 10^{7} \mathrm{HZ} / \mathrm{T}$. Recalling once again the most important fact that only excess nuclei of low energy state (spin up) are responsible for MR signal generation in the presence of external magnetic field $\mathbf{B}$ and their magnetic moments $\boldsymbol{\mu}$ revolves around $\mathbf{B}$ with angular moment $\mathbf{J}$ as shown in Figure $7(\mathrm{~b})$ and the relation is expressed in Equation (1) and Equation (2). As a result the net magnetization vector $\mathbf{M}$ is formed in the direction of $\mathbf{B}$. The resultant $\mathbf{M}$ related to resultant angular moment $\mathbf{J}$ is given by Equation (3). Recalling once again the most important fact that only excess nuclei of low energy state (spin up) are responsible for MR signal generation in the presence of external magnetic field $\mathbf{B}$ and their magnetic moments $\boldsymbol{\mu}$ revolves around $\mathbf{B}$, as a result the net magnetization vector $\mathbf{M}$ is formed in the direction of $\mathbf{B}$.

$$
\begin{gathered}
\gamma=\boldsymbol{\mu} / \boldsymbol{J} \\
\boldsymbol{\mu}=\gamma \boldsymbol{J} \\
\boldsymbol{M}=\gamma \boldsymbol{J}
\end{gathered}
$$

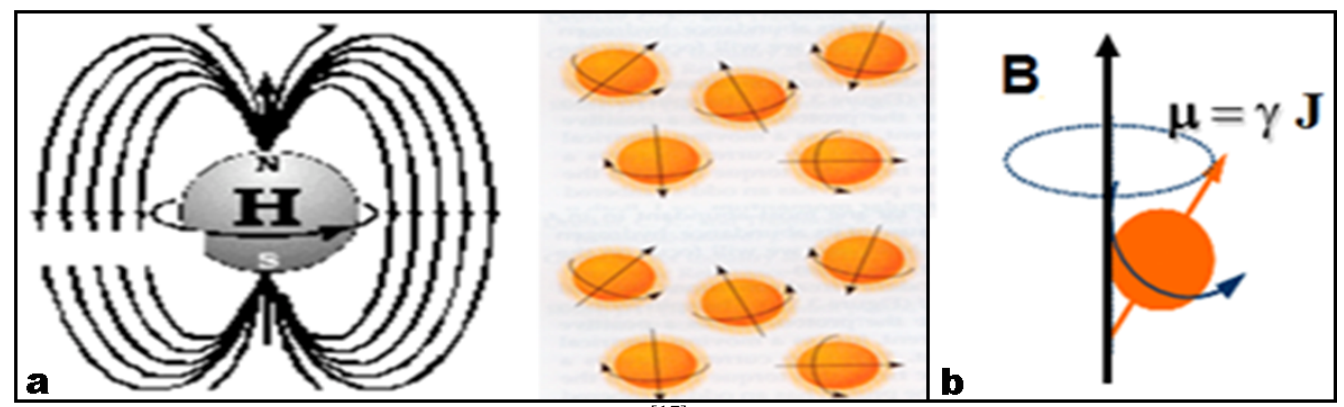

Figure 7. (a) Proton spin produces magnetic dipole ${ }^{[17]}$; (b) Angular moment $\mathbf{J}$ and Magnetic moment $\boldsymbol{\mu}^{[18]}$. 


\subsection{Larmor frequency}

The magnetic moment $\mathbf{M}$ produces net angular torque $\mathbf{J}$ which can be expressed in the form of vector product in Equation (4) as follows:

$$
\boldsymbol{J}=\boldsymbol{M} \times \boldsymbol{H}
$$

Where $\mathbf{H}$ is strength of applied magnetic field $\mathbf{B}$ and $\mathbf{M}$ is net Magnetization of spin system. Due to torque $\mathbf{J}$, the magnetization vector $\mathbf{M}$ is not at fixed position in space instead it is continuously moving in a gyrating fashion around the axis of $\mathbf{B}$. The classical analogy to this phenomenon is the spinning behavior of top in gravitational field where precession axis represents the axis of applied magnetic field $\mathbf{B}$ and spin axis is the direction of tiny magnetic moments vector $\boldsymbol{\mu}$. The rate of change of $\mathbf{J}$ w.r.t time is expressed in Equation (5) whose little manipulation yields frequency in Equation (6):

$$
\begin{gathered}
\frac{\partial \boldsymbol{J}}{\partial t}=\frac{\partial(\boldsymbol{M} X \boldsymbol{H})}{\partial t} \\
\frac{\partial(\gamma \boldsymbol{J})}{\partial t}=\frac{\partial}{\partial t} \gamma(\boldsymbol{M} X \boldsymbol{H}) \\
\frac{\partial \boldsymbol{M}}{\partial t}=\gamma \frac{\partial}{\partial t}(\boldsymbol{M} X \boldsymbol{H}) \\
\omega=\gamma \boldsymbol{B}
\end{gathered}
$$

Hence for a static magnetic field $\boldsymbol{B}$ with strength $\mathbf{H}$, all spins precess at same rate $(\omega=\boldsymbol{\gamma} \boldsymbol{B})$ but if somehow the strength of field $\mathbf{B}$ is made non-uniform for example by external excitation different protons may precess at different frequencies. This $\omega$ is the frequency at which magnetization vector $\mathbf{M}$ precess in external magnetic field $\mathbf{B}$ and is called as Larmor frequency, or processional frequency or resonant frequency ${ }^{[1,2]}$. Since vector $\mathbf{M}$ rotates about longitudinal z-axis the component $M_{z}$ remains same while components $M_{x}$ and $M_{y}$ changes with time as shown in Figure 8 . The two ways to look at this rotation is: (1) $\mathbf{M}$ or net magnetic moment $<\boldsymbol{\mu}>$ rotates in 3D spaces, called as lab frame; (2) $\mathbf{M}$ or net magnetic moment $\langle\boldsymbol{\mu}\rangle$ is stationary and the frame of reference is in rotation, called as rotating frame.

Figure 8. Rotation of Magnetization vector $\mathrm{M}$ or net magnetic moment $<\mu>$ about $\mathrm{z}-$ axis; Magnetization vector in rotating frame $x^{\prime} y^{\prime}$. The picture is adapted from ${ }^{[20]}$.

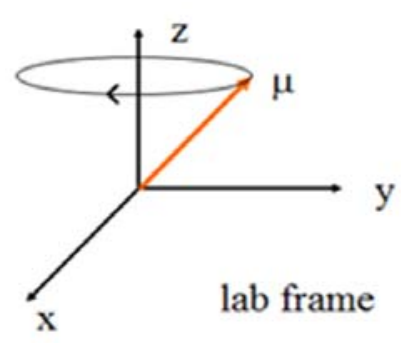

\subsection{Polarization}

The randomly oriented magnetic moments $\boldsymbol{\mu}$ changes their orientation when they are subjected under a high and stable magnetic field of strength 1 to 4 Tesla or more. In the presence of such a strong magnetic field, the protons spin vectors will align themselves in either parallel or anti-parallel fashion to the applied magnetic field $\mathbf{B}$. This is exactly the first main magnetic field that a patient experience in MRI scanner as shown in Figure 9. We represent the applied magnetic field with symbol $\mathbf{B}$ which is proportional to the magnetic susceptibility $\mathbf{H}$ of protons. $\mathbf{B}$ and $\mathbf{H}$ are related to each other by $\boldsymbol{\chi}$, the free space permeability of space and the relation is given in Equation (7):

$$
\boldsymbol{B}=\chi \boldsymbol{H}
$$

The quantity $\mathbf{B}$ is usual magnetic flux density with dimensions $\left(\frac{W b}{m^{2}}\right)$, where $W b=$ Ampere - Henry $(A H)$. 

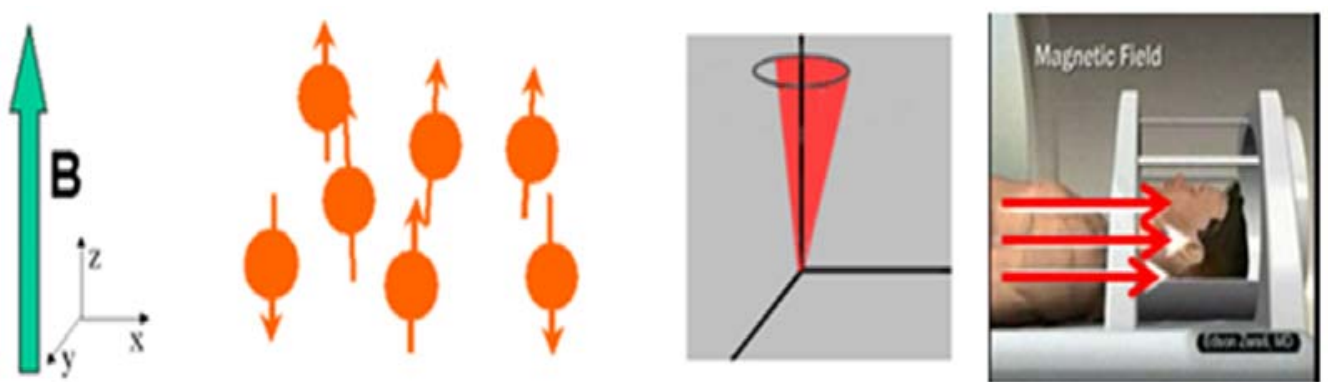

Figure 9. Spins aligned with B (Spin Up) while Spins $180^{\circ}$ out of phase with B (Spin Down). The picture is adapted from ${ }^{[16]}$.

The Orange spheres in Figure 9 represents $\boldsymbol{\mu}$ (magnetic moment) vectors parallel or aligned with $\mathbf{B}$ and are in state of lower energy "spin up", while some spheres represent the spin that are $180^{\circ}$ out of phase with $\mathbf{B}$ and are in a higher state of energy "spin down". The basic Quantum mechanics facts to be used here are:

(1) Not every isotope excites and establishes new energy levels. For e.g. Carbon ${ }_{6}^{12} \mathrm{C}$ and Oxygen ${ }_{8}^{16} \mathrm{O}$ will not produce new energy levels when excited by external magnetic field.

(2) When an external field is applied on nucleus to excite, the energy levels only exist in discrete or quantum state. Spin number $=-j,-j+1, \ldots j-1$.

(3) "Spin up" and "spin down" are basically two energy levels established by hydrogen ${ }_{1}^{1} H$ when external magnetic field of strength $\mathbf{B}$ is applied to it see Figure 10. The spin number $j$ for hydrogen is $\frac{1}{2}$ or $-\frac{1}{2}$. Let the concentration of Spin down nuclei is represented by $\mathrm{N}+$ and the concentration of Spin up nuclei be $\mathrm{N}$ - as it is the only excess nuclei of lower energy state (spin up) that generate MR signal. Further let that $\Delta N$ be the difference between these two concentrations and is governed by Boltzmann distribution.

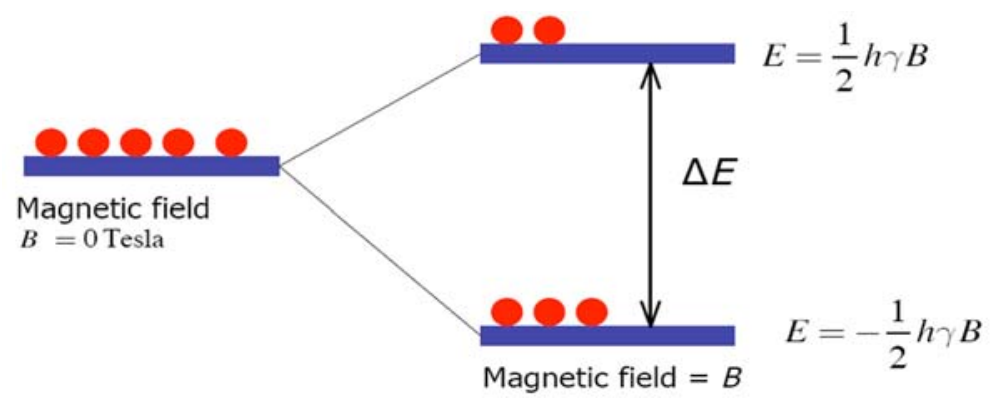

Figure 10. Hydrogen influenced by Magnetic field B with spin number ${ }^{1 / 2}{ }^{[17]}$.

So First we determine $\Delta N$ and then with the help of $\Delta N$, we figure out the net magnetic dipole $\boldsymbol{\mu}$ and net magnetization $\mathbf{M}$ in the magnetic field of strength $\mathbf{B}$. According to Boltzmann law, the equilibrium ratio between two states of different energy $(\Delta \mathrm{E})$ is given by $e^{-\Delta E / k T}$. Hence as per Boltzmann's distribution:

$$
\frac{N+}{N-}=e^{-\Delta E / k T}
$$

$\mathrm{N}+=$ concentration of spin down nuclei (high energy state); $\mathrm{N}-=$ concentration of spin up nuclei (low energy state); $\mathrm{T}=$ normal body temperature in kelvin $=310 K ; k=$ Boltzmans constant $\left(8.26 \mathrm{e}^{-5} \mathrm{ev} / K\right.$ or $\left.1.38 \mathrm{e}^{-23} \mathrm{~J} / K\right) ; \Delta \mathrm{E}=h \vartheta=h \frac{\omega}{2 \pi}=$ $\frac{h}{2 \pi}(\gamma \boldsymbol{B})$.

The difference in energy between two states is labeled $\Delta E$ in Figure 10 and the parameters involved in $\Delta E$ expression are summarized below: 
$\mathrm{h}=$ Planck's Constant $=6.63 \mathrm{e}^{-34} J S=4.14 \mathrm{e}^{-15} \mathrm{eV} ; \vartheta$ and $\omega$ are frequency in $\mathrm{Hz}$ and $\mathrm{rad} / \mathrm{sec}$ respectively; $\gamma$ is gyromagnetic ratio; B is strength of applied magnetic field in $\mathrm{Wb} / \mathrm{m}^{2}$.

Listed below are the facts to simplify $\Delta E$ Equation, so that we get $\Delta N$ :

- Taylor expansion for exponential is applied on Equation (8) and only first two terms are kept as value of exponential is getting smaller and smaller;

- $\quad \mathrm{N}+$ and $\mathrm{N}-$ are approximately same;

- $\quad \mathrm{N}+=$ half of total nuclei $N_{T}$.

Equation (8) becomes:

$$
\frac{N+}{N-} \approx 1-\frac{\Delta E}{K T}
$$

Now using:

$$
\begin{gathered}
\Delta N=(N-)-(N+)=\frac{\Delta E}{k T} N+\approx \frac{\Delta E}{k T} \frac{1}{2} N_{T} \\
\Delta N=\frac{h}{2 \pi} \gamma \boldsymbol{B} \frac{1}{2} \frac{N_{T}}{k T}
\end{gathered}
$$

When we multiply this excess numbers of nuclei $\Delta N$ with the average of magnetic moments $\boldsymbol{\mu}$, we will get net magnetic dipole moment $\mathbf{m}$ and magnetic dipole per unit volume $\mathrm{V}$ so it defines required magnetization signal $\mathbf{M}$ as follows with illustration in Figure 11:

$$
\boldsymbol{m}=\Delta N\langle\boldsymbol{\mu}\rangle ; \boldsymbol{M}=\frac{\boldsymbol{m}}{\boldsymbol{V}}
$$

Figure 11. Magnetization signal $\mathbf{M}$ due to spin up protons ${ }^{[18]}$.

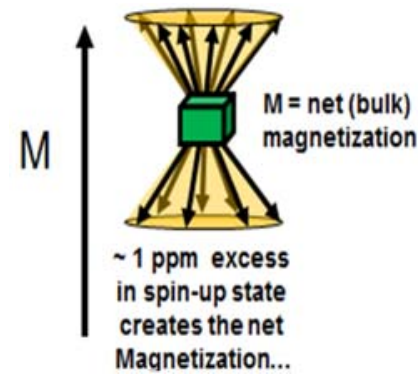

\subsection{Bloch equation}

The key discovery in the field of MRI imaging is attained by Professor Bloch ${ }^{[1]}$ who was primarily interested in the time variation of vector $\mathbf{M}$ as this nuclear magnetization $\mathbf{M}(\mathrm{t})$ is the source of "NMR signal" from which the image is ultimately constructed. He excite the spin system using Radio frequency (RF) waves of frequency near to resonant frequency $\omega_{0}$ and then allow spins to relax (decay) to measure $\mathbf{M}(\mathrm{t})$. The mathematical model of Bloch equation as black box whose input is magnetic field strength $\mathbf{H}(\mathrm{t})$ and whose output is $\mathbf{M}(\mathrm{t})$ is depicted in ${ }^{[19]}$. The black box depiction is illustrated in Figure 12 followed by description of system parameters $M_{0}, T_{1}$ and $T_{2}$ as follows:

Figure 12. Black box depiction of bloch equation.

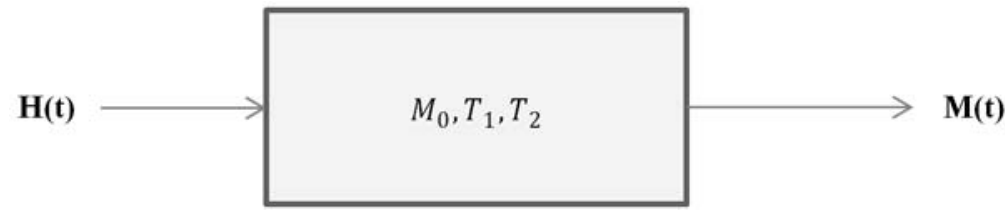

ISSN 1925-4008 E-ISSN 1925-4016 
$M_{0}$, is the z-component of magnetization vector $\mathbf{M}$ written as $M_{0} \boldsymbol{k}$ and represents the thermal equilibrium state i.e. when in the presence of external magnetic field $\boldsymbol{H}$ there is no signal emitted by protons and their spins are lined up with $\boldsymbol{H}$ in parallel or anti parallel fashion as discussed before and is expressed as follows in Equation (12):

$$
M_{0}=\chi H_{0}
$$

Where $\chi$ is the nuclear paramagnetic susceptibility.

$T_{1}$ called "Thermal", "longitudinal" or "spin-lattice", relaxation time in literature ${ }^{[1,19]}$ is a constant. This is the time when in the presence of external magnetic field $\mathbf{H}$, nuclei spin system thermal equilibrium has been knocked by RF excitation for a moment, so $M_{z} \neq M_{0}$ and excited protons from high energy state (spin down) tends to attain their stable low energy state (spin up) and thus generate a signal. The change in magnetization vector $\mathbf{M}$ w.r.t time $t$ is expressed in differential form in Equation (13):

$$
\dot{M}(t)=\frac{\left(M_{z}-M_{0}\right) \boldsymbol{k}}{T_{1}}
$$

Figure 13 shows different spin states. When RF excitation is applied at $90^{\circ}$ to main magnetic field $\mathbf{B}$ with strength $\mathbf{H}$, the protons attain thermal equilibrium after certain time; means are either aligned parallel or anti parallel with $\mathbf{B}$ with no signal at all. When RF excitation is removed at $\mathrm{t}=0$, the proton spins and in turn net magnetization component $M_{z}$ fall to zero in no time and then rises exponentially to attain thermal equilibrium again. $T_{1}$ is the time when $M_{z}$ reaches $63 \%$ of equilibrium value.

Figure 13. Exponential recovery of magnetization $\boldsymbol{M}_{\mathbf{z}}$ versus $\boldsymbol{T}_{\mathbf{1}}$ (longitudinal relaxation time). The Picture is adapted from ${ }^{[17]}$.

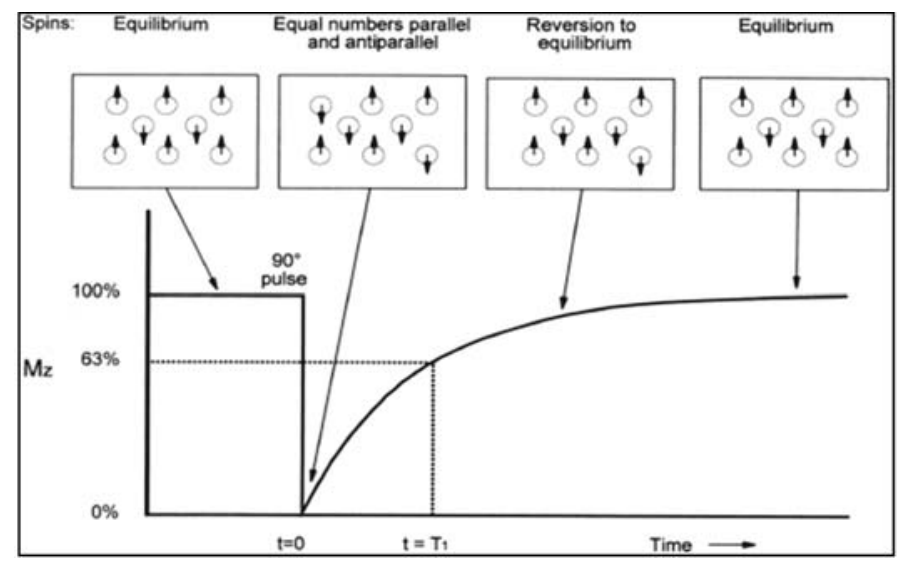

$T_{2}$, "transverse" or "spin-spin" relaxation time is also a constant. This depicts the time taken by nuclei spins to orient them randomly or to make the value of net magnetization vector $\mathbf{M}$ as zero. Recall this happens when there is no external magnetic field $\mathbf{H}$. The change in magnetization is given by following differential equation:

$$
\dot{M}=\frac{M_{x} i+M_{y} \boldsymbol{j}}{T_{2}}
$$

Figure 14 depicts the situation when at $\mathrm{t}=0, \mathrm{RF}$ is switched off and the transverse component $M_{x y}$ starts decaying exponentially to zero in contrast to longitudinal component which recovers itself from zero to $M_{z}$.

The complete Block equation for magnetization $\mathbf{M}$ using Equation (3), Equation (13) and Equation (14) is thus written as following differential equation:

$$
\dot{M}(t)=\gamma[\boldsymbol{M} \times \boldsymbol{H}]-\frac{M_{x} \boldsymbol{i}+M_{y} \boldsymbol{j}}{T_{2}}-\frac{\left(M_{z}-M_{0}\right) \boldsymbol{k}}{T_{1}}
$$


Figure 14. Exponential decay of Magnetization $\boldsymbol{M}_{\boldsymbol{x} \boldsymbol{y}}$ versus Transverse relaxation time $\boldsymbol{T}_{\mathbf{2}}$ The Picture is adapted from ${ }^{[17]}$.

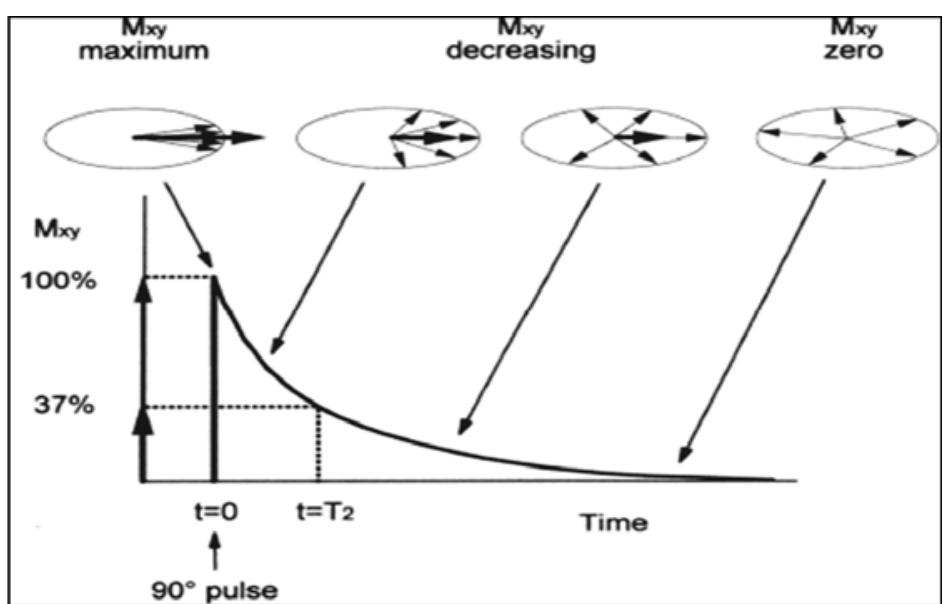

Transverse component $M_{x y}$ thus rotates about longitudinal (z-axis) with precession frequency $\omega_{0}$ as shown in Figure 15 . This will induce current in a coil placed perpendicular to transverse plane due to precession $\mathbf{M}$ whose rotation can be written in matrix form as follows:

$$
\left[\begin{array}{c}
M_{x}(t) \\
M_{y}(t) \\
1
\end{array}\right]=\left[\begin{array}{ccc}
e^{-t / T_{2}} & 0 & 0 \\
0 & e^{-t / T_{2}} & 0 \\
0 & 0 & 1
\end{array}\right]\left[\begin{array}{ccc}
\cos \omega t & -\sin \omega t & 0 \\
\sin \omega t & \cos \omega t & 0 \\
0 & 0 & 1
\end{array}\right]\left[\begin{array}{c}
M_{x}^{0} \\
M_{y}^{0} \\
1
\end{array}\right]
$$
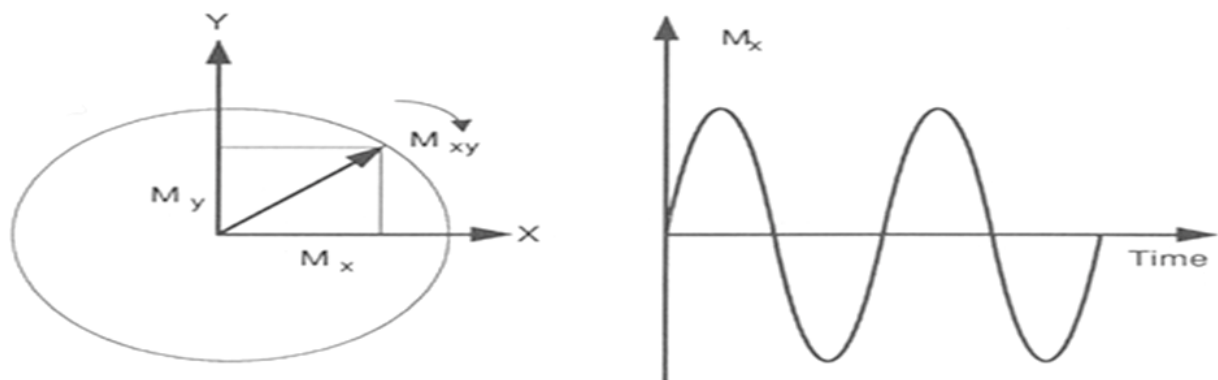

Figure 15. Rotation of transverse magnetization component about longitudinal axis and Induced Voltage in a coil aligned along the $\mathrm{Y}$-axis as a function of time ${ }^{[20]}$.

The longitudinal component initial values are $M_{z}^{0}$ and its final value is its equilibrium value represented by $M_{0}$. Thus $M_{z}(t)$ can be written as linear combination of these two values as follows:

$$
\begin{gathered}
M_{z}(t)=M_{z}^{0} t+M_{0}(1-t) \\
M_{z}(t)=M_{z}^{0} e^{-t / T_{1}}+M_{0}\left(1-e^{-t / T_{1}}\right)
\end{gathered}
$$

The two relaxation times $T_{1}, T_{2}$ and the change in magnetization can be visualized in Figure 16.

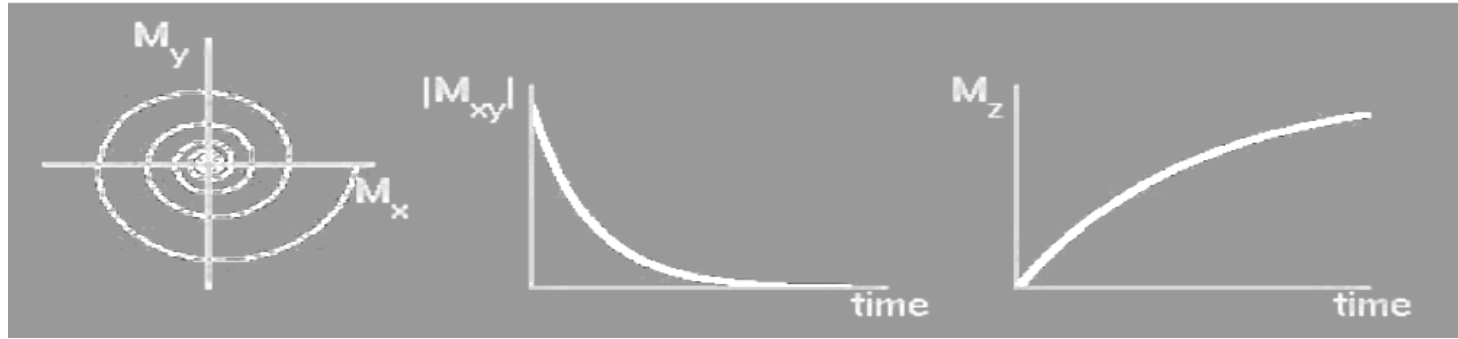

Figure 16. Rotation, decay and recovery of magnetization components. The picture is adapted from ${ }^{[21]}$. 


\section{I mage formation}

The signal that is produced in coil placed near by rotating magnetization vector $\mathbf{M}$ perpendicular to transverse plane is characterized by its amplitude, phase and frequency. The MR Image formation process comprises of (1) Slice Selection; (2) Phase encoding; (3) Frequency encoding, Read Out Phase; (4) K-Space Matrix; (5) Inverse Fourier Transform. A Slice from human subject is selected by controlling the frequency $\omega$ of the spins. The initially applied $90^{\circ} \mathrm{RF}$ pulse enforce all protons to spin at same resonant frequency but the application of linearly varying gradient field $G$ causes protons within selected slice to spin at different frequency. The technique of making frequency $\omega$ to depend on position of gradient is the key to image formation. For slice selection only z-gradient $G_{z}$ is kept on, as a result the modified equation for frequency $\omega$ becomes:

$$
\omega=\gamma\left(M_{0}+z G_{z}\right)
$$

Substituting $\omega=2 \pi f_{c}$ we have:

$$
\begin{gathered}
f_{c}=\frac{\gamma\left(M_{0}+z_{c} G_{z}\right)}{2 \pi}=\frac{\gamma M_{0}}{2 \pi}+\frac{\gamma z_{c} G_{z}}{2 \pi}=f_{0}+\frac{\gamma z_{c} G_{z}}{2 \pi} \\
\Delta f=\frac{\gamma \Delta z G_{z}}{2 \pi}
\end{gathered}
$$

Where $\Delta z$ represents the thickness of slice centered at $z_{c}$ with all protons in it spins at frequency $f_{c}$ as shown in Figure 17. Note that greater bandwidth means $\Delta f$ increases as a result slice thickness increases.

Figure 17. Slice Selection by applying linearly varying gradient field along z-axis. Picture is taken from ${ }^{[22]}$.

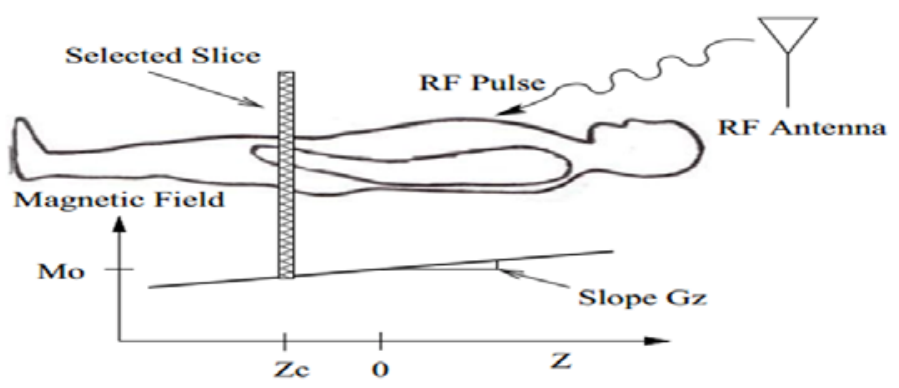

The RF pulse in time domain is a sinc function whose frequency domain representation is simply a rectangular pulse given in Equation (19) as follows.

$$
s(t)=e^{j 2 \pi f_{c} t} \operatorname{sinc}(t \Delta f) \leftrightarrow S(f)=\operatorname{rect}\left(\frac{f-f_{c}}{\Delta f}\right)
$$

For 2D imaging the magnetic gradients are now allowed to varied along both $\mathrm{x}$ and $\mathrm{y}$ axis. In the meantime $\mathrm{z}$ gradient is switched off and thus protons emit RF energy with location dependent frequency. This emitted RF energy is measured and is used to reconstruct 2D image. The image formation process of MRI is illustrated in Figure 18 followed by its explanation.

(1) Human subject is placed in static strong magnetic field $\mathbf{B}$ with strength $\mathbf{H}$, the randomly oriented protons spin becomes aligned with $\mathbf{B}$ and attains thermal equilibrium.

(2) Protons are allowed to enter into excited state by providing RF energy of particular frequency via additional gradient coils that changes existing magnetic field $\mathbf{B}$ and thus perturb equilibrium. The gradient also makes the protons frequency to be dependent on position.

(3) The Protons are then allowed to relax means they emit the RF energy of location dependent frequency and try to attain the same thermal equilibrium state. 
(4) The RF signal is detected and image is reconstructed via slice selection, phase encoding, and frequency encoding and taking inverse Fourier transform of K-Space data. The details of these steps are beyond the scope of our work.

Figure 18. MR Image formation summary ${ }^{[17]}$.

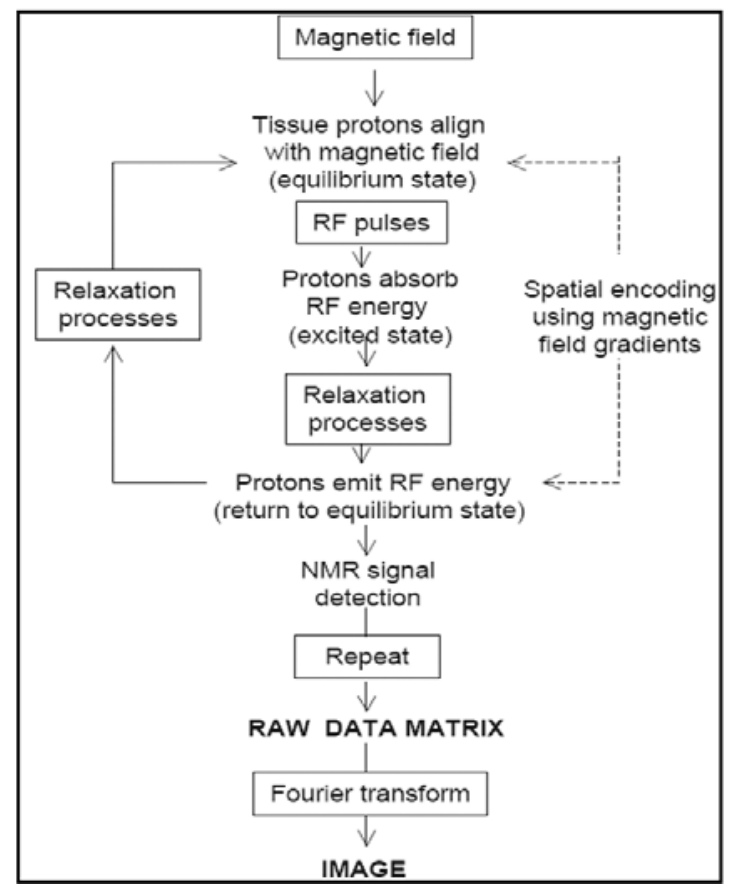

\section{Conclusion}

The review would be beneficial for acquiring study background for interdisciplinary research especially in the field of MRI image processing. The glorious history of MRI itself creates a motivational momentum for understanding relevant concepts and is part of this review. MRI image processing is a field that blends biomedical sciences, neuro-sciences and computer sciences amazingly. The visualization process of brain MR slices encompasses worthy concepts of coordinate systems and camera analogy from computer graphics domain. This process of $3 \mathrm{D}$ visualization is accompanied by strong statistical analysis and image processing concepts. The brain MR image processing, qualitative and quantitative interpretations pushes research work towards the field of neuroimaging. Thus an understanding and review of spin physics, image formation, fourier transform and coordinate space understanding is a corner stone to relate various domains.

\section{Conflict of interests}

The authors declare that they have no conflict of interests.

\section{References}

[1] Bloch F. Nuclear Induction. Phys. Rev. 1946; 460: 474. http://dx.doi.org/10.1103/PhysRev.70.474

[2] Purcell E, Torry H, Pound R. Resonance absorption by nuclear magnetic moments in a solid. Phys Rev. 1946; 69: 37. http://dx.doi.org/10.1103/PhysRev.69.37

[3] Geva T. Magnetic Resonance Imaging: Historical Perspective. Journal of Cardiovascular Magnetic Resonance. 2006 ; 573 : 580.

[4] Creasy J L. The Structure of the Normal Brain and Its Imaging Appearance. Dating Neurological Injury. New York, Springer. 2011; 3: 41.

[5] Clow H, Young I. Britain's brains produce first NMR scans. New Scientist. 1978; 80: 588.

[6] Godfrey N. Computed Medical Imaging. Nobel Lectures in Physiology and Medicine. 1979; 568: 586. 
[7] Gregor T O. Exploration of Human Brain Tissue. Micscape Magazine [Internet]. October 2002; 84. Available from: http://www.microscopy-uk.org.uk/mag/issueindex.html

[8] Elva O' S. In the Human Body [Internet]. 2012. Available from: http://www.sciencewithme.com

[9] Tsai C, Manjunath B, Jagadeesan R. Automated Segmentation of Brain MR Images. Pattern Recognition. 1995; 28(12): 1825: 1837.

[10] Atkins M, Blair T M. Fully Automatic Segmentation of the Brain in MRI: IEEE Transactions on Medical Imaging. 1998; 17, 98: 106.

[11] Shattuck W D, Stephanie R, Kirt A S, Rottenberg D A, Leahy R M. Magnetic Resonance Image Tissue Classification Using a Partial Volume Model. NeuroImage. 2001; 856: 876.

[12] Neuroscience: The Brain [Internet]. Available from: http://eweb.furman.edu/ einstein/general/neurodemo/96B.gif

[13] Goldman S A. The merck manual home health hand book. November 2007. Available from: http://www.merckmanuals.com/home/brain_spinal_cord_and_nerve_disorders/biology_of_the_nervous_system/brain.html.

[14] Nobelprize.org [Internet]. Available from: http://www.nobelprize.org/nobel_prizes/physics/laureates/1901/perspectives.html.

[15] Jerrold T B, Seibert J A, Edwin M L, Boone J M. The Essential Physics of Medical Imaging. 2003.

[16] Hornak J P. The Basics of MRI: Henietta, NY, Interactive Learning Software. 1996-2014.

[17] Kinahan P. Bioengineering 508[Internet]. 2006. Available from: http://courses.washington.edu/bioen508/

[18] Schillaci M J. An Introduction to MRI Physics and Analysis. Columbia. 2008.

[19] Waldo S H, Arnold H L. An Introduction to NMR Imaging. From the Bloch Equation to the Imaging Equation. 1983.

[20] Shung K K, Benjamin M S, Tsui M. Principles of Medical Imaging. California: Academic Press. 1992.

[21] Bloch Equation Simulation [Internet]. Available from: http://mrsrl.stanford.edu/ brian/bloch/

[22] Bouman C A. EE637: Digital Image Processing I, Purdue University [Internet]. 2014. Available from: https://engineering.purdue.edu/ bouman/ece637/notes/ 